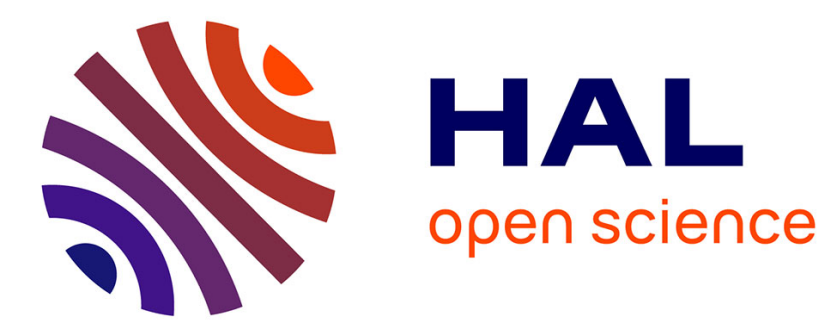

\title{
L'Usage de la 3D en Archéologie
}

Robert Vergnieux

\section{To cite this version:}

Robert Vergnieux. L'Usage de la 3D en Archéologie. Informatique et Egyptologie, Nigel Strudwick, Jul 2008, Vienne, Autriche. pp.147-154. hal-02138058

\section{HAL Id: hal-02138058 https://hal.science/hal-02138058}

Submitted on 29 May 2019

HAL is a multi-disciplinary open access archive for the deposit and dissemination of scientific research documents, whether they are published or not. The documents may come from teaching and research institutions in France or abroad, or from public or private research centers.
L'archive ouverte pluridisciplinaire HAL, est destinée au dépôt et à la diffusion de documents scientifiques de niveau recherche, publiés ou non, émanant des établissements d'enseignement et de recherche français ou étrangers, des laboratoires publics ou privés. 
Eibue in TExhnotact4 $\mathrm{z}$

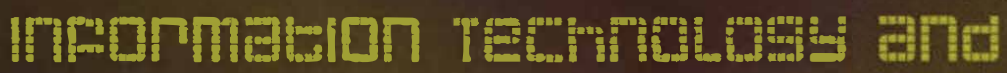

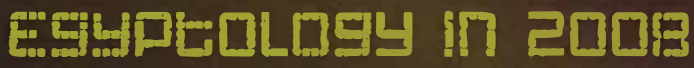

Proceedings of the meeting of the Computer Working Group of the International Association of Egyptologists (Informatique et Egyptologie), Vienna, 8-11 July 2008 


\title{
L'USAGE DE LA 3D EN ARCHÉOLOGIE
}

\author{
Robert Vergnieux
}

\section{ABSTRACT}

The ase of 3D modeling in antbaeology bas developed fromt the struightforsurd production of images for illustratule purposes into becoming an effective tool to aid scientific archaeological research Virtwal Reality allows not onty the restoration of ancient struttures abich bave now disappeared but also permits the testing of new bypotheses as to bow these strutures worked. A particular use within Egyptology bas been the non-invasive studies of mummies with this new technoloyy. Howezer; in ziew of the importance of architectural remains along the Nile ealley, there can be little doubt that the most significant potential lies in the virtual restoration of those stractures Although $3 D$ images allow the public to wnderstand better these processes, se should never lose sight of the fact that theer production was driven by undertying scientific goals $A$ 3D tecbnological plafform specifically for work suth our architec-zural hertage has been created in Bordeawx; th is especrally adapted for 3D scanning, modelling, and maintaining the persistence of $3 D$ digital data

\section{UNE PLATE-FORME TECHNOLOGIQUE 3D}

L'UMR 5607 du CNRS s'est doté d'une espace architectural complétant la Maison de l'Archéologie. Un nouveau bâtiment de près de mille mètres carrés, se compose de salles "chercheurs", d'un laboratoire de céramologie, d'un espace d'exposition et d'une Plate-forme Technologique 3D du CNRS. I.e concept de ce bâtiment repose sur la possibilité donnée au public de venir voir la recherche en archéologie "en train de se faire ». L'archéopôle n'est pas un muséc mais plutôt une interface entre les citoyens et les chercheurs. 
L'imagerie 3D envahie aussi bien les programmes de recherches en archéologie que les médias. Dans ce contexte, l'un des enjeux majeurs de la Plate-forme Technologique 3D de l'Institut Ausonius est de promouvoir auprès des équipes de recherche l'utilisation des technologies de la $3 \mathrm{D}$ en archéologique en tant qu'ouril de recherche et non pas seulement comme un moyen d'illustration. Les modèles 3D conçus dans le cadre de la PFT3D sont à la disposition de la communauté des chercheurs tout en restant sous le control des équipes qui les ont élaborés.

La PFT3D dispose d'une plate de production de neuf postes de développement 3D, et d'un poste de scannographie laser 3D. L'ensemble des données est géré localement à l'aide de deux serveurs Raid 5 (DELL) et de deux disques de sauvegarde d'un téraoctet chacun. La salle réalité virtuelle (odéon) permet, lors des séminaires de validation des restitutions 3D d'afficher sur un écran de $8,5 \times 3,5$ des scénes $3 \mathrm{D}$ de 2496 pixels $\times 1050$ pixels à l'aide d'un cluster de PC. L'odéon sert également pour la présentation au public des dossiers de recherche. Sa capacité est de 96 places.

Toutes les données produites par la PFI3D sont archivées et pérennisées dans le Conservatoire National des Données Numériques 3D du patrimoine que nous avons crée.

\section{MÉTHOdOLOGIE PARTICULIÉRE DE LA MISE EN GEUVRE DES RESTITUTIONS 3D}

Par restitution nous entendons l'acte scientifique de restituer, à une époque ou date précises, des structures antiques disparues ou dégradées, et non pas le fair de transposer en numérique des édifices encore existants du patrimoine.

\section{a) mise en place d'un modẻle préparatoire - niveau V1}

I $e$ travail de restitution d'un site archéologique majeur est un travail d'équipe et ne peut plus être le fait d'un seul individu. L'élaboration d'un modèle numérique $3 \mathrm{D}$ requiert des compétences spécifiques de plusieurs natures. Des connaissances archéologiques et historiques sur le site étudié sont nécessaires tout autant que la connaissance du site : topographie des vestiges encore en place, caractérisation des matériaux, données archéologiques de toute nature (archéométrie).

La difficulté de mise en ceuvre de tels projets pluridisciplinaires réside dans la façon de faire dialoguer tous les spécialistes entre eux d'une part et 
d'arriver à engrancer les avancés signiticatives en termes de validation des restitutions des espaces antiques. C"est précisément ici que le madele numć rique 3D revêt toute son importance. Tant qu'un modèlc '3D n'es? pas visualisable, lo dialogue critre spécialistes est délicat, chacun des scientifiques ayant sa propre vision des valumes. Dès qu'une première ébatiche tridimensionnelle est visualisable alors il devient possible a deux chercheurs de champs disciplinaires óstincts de dialoguer entre sux sur la testitution de "volumes" disparus. Une arpumenwaion peut se raetrate en place de faças. précise chacun activan ses connaissances ropres face aux démils de la rostatutien 3D visualisable par tous. La toute première étape des projots

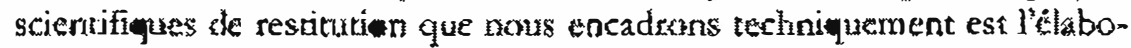
ration incontournable de cette prenière ébauche 3D des édifices étudies tenant coripe des eyentuelles hypothèses antérrieures si elles existent.

Ces modèles à cette étape ae sont certes pas encore validés scienciftquernent mais ils sont indispensables pour la mise era place d'un dialogute entre tous les partenaires scientificues Nous appelons ce niveau de yersion: V1.

\section{b) Organisation de la documentation nécessaire au travail de restitution $3 \mathrm{D}$}

C'est parällèlement â certe première téalistation que nous constiturans la base documentaire de l'ensemble des serurces qui seront néressaires á l'opération de restitution.

- Données épigraphiques

Attestations des norns des édifices

Attestations textuelles d'existence de bäriments

Attestztions extuellex d'éyènements liés à des báciments

- Données iconographiques avec la représentadion d'édifices

- Vestiges archéclogienues de terrains (epars or in siru)

- Hzpothèses antrérieures de restiturion (nxaquettes physiques, dessins, modèles numériques)

- Documents complementaires (paralleles decumentaires possible er issus d'édifices d'sutres périodesis)

Dans te cadre de la PFT3D nous suons min au point une interface spécifigue de manipulation de ces donnés, sorte de table lumineuse yirnuelle (tabö̈de) accessible à rous les participants quelque soir lcur localisatisn géographique. La partic "Gactuelle» de ces données est donc mise en 
commun. Chaque chercheur peut y adjoindre ses propres informations s'il le souhaite. Ie tablö̈de utilise également une structure particulière d'« unicos » et d'، unitexte ». Ce sont des fragments d'image ou de texte issus d'un document permettant la manipulation du contenu sémantique des images et des textes (Vergnieux 1999).

\section{c) Séances de validation des restitutions}

Nous organiserons des séminaires autour d'un ordre du jour précis lié à la restitution des édifices concernés. Les personnes présentes lors de ces séminaires disposent donc d'une "scène 3D " manipulable en temps réel permettant de visualiser collectivemenr n'importe quel secteur et dérail 3D du site en cours d'étude. Les personnes présentes ont également accès pendant le séminaire à l'ensemble des sources liées au projet de recherche. Nous pouvons inviter ponctuellement, lors d'une séance, un chercheur ayant des compétences particulières liées à l'ordre du jour.

Chaque séminaire a pour conséquence de faire évoluer la connaissance et les hypothèses de restitution. Il est donc nécessaire d'actualiser le modèle numérique $3 \mathrm{D}$ en fonction des séminaires. Les différenres versions sont qualifiées de second niveau (versions $V 2 . x$ ). Ies séminaires se tiennent autanr de fois que possible et nécessaire faisant évoluer les modèles d'unc version $V 2 x_{x}$ en une version $V 2 \cdot(x+1)$. Lors de ces séminaires il arrive aussi de découvrir de nouveaux documents qui sont alors ajoutés dans la base documentaire des différenrs corpus qui s'accroît régulièrement en quantité et en qualité. Enfin des liens entre les modèles numériques 3D et les sources documentaires reposent sur le concept de nomenclature 3D. C'esr à dire que pour l'ensemble des édifices du règne, nous définissons un vocabulaire commun décrivant la hiérarchic "volumique » de tous les sires archéologiques étudiés dans ce projet. Cette nomenclature conscitue l'arête dorsale du projet après sa validacion par les membres de l'équipe. A parcir d'un élément quelconque du modèle numérique il devient alors possible d'interroger les corpus documentaires associés.

L'ensemble du travail fait selon la méthode indiquée amène progressivement à la construction de modèles numériques en version V3. Versions dont les membres du projet s'accordent à dire qu'elles sont conformes aux hypothèses scicntifiques actuelles. I es modèles en version V3 sont destinés aussi à évoluer en fonction de nouvelles avancées de la recherche. Cependant ils sont d'un niveau suffisamment avancé pour servir de support à la communication scientifique ainsi qu'à la communication vers le public au titre de la valorisation des programmes de recherche. 


\section{L'EGYPTOLOGIE ET L'USAGE DE LA 3D POUK LA RESTITUTION DES ÉDIFICES AUJOURD'KUX DISPARUS}

Pour visualiser les yes inges pharaoniques, l'usage de maquettes en « dur \# est encore assez courant taf elles renconttent toujours un grand succès auprès du public. Comme prut les aumes domaines de l'archétologie, c'est a l'occasion de la réalisation de films documentaxires ou d'expusitions qu’apparaissent des modéles 3D de resitutions. Rare sont les équipes ézýp' tologiques utilisant les mrideles 3D pour effectuer des recherches dans le sens des procédures décrites ci-dessus. Les modeles 3D se limitent le plus souvent ane fonction dillustration. Il faut rappeler ici le projet pionatier dans lequel dès 1986 des équipes mixtes d'archéolż̧̨ıes et Ingénieurs ont collabore à la réalisamon d'un modèle numérique des temples de Karnak (Alberuy et al 1989). Ce projet pilote avait ouvert la voie à l'usaz̧e de l'image de syrthèses entant que support pour la recherche de restitution. I. e parenciel des modèles numéziques 3D est actuellement sous utilisé. Enxtore ptésenté comme une illustration construite en fin de prsjet, les mexdèles numériques $3 \mathrm{D}$ prenoent cependaot tout leur sens s'ils sont introduits en début de recherche sar l'actionde restituer les architectures disparuess selève de véritables prozyrarames pluridisciplinaites.

C'est à trivers de tels ptojets qu'il devient possible de mieux comprendre les bấtiments construits par les anciens, de mieux comprendre keur fonctionnement et ainsi de mieux comprendre les socictés qui les ont érigés.

\section{REFERENCES}

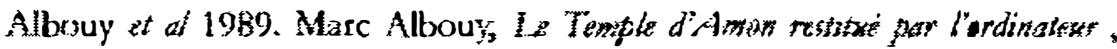
Solar, 1999.

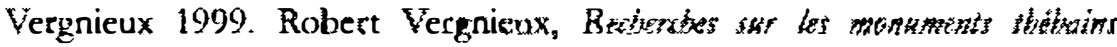

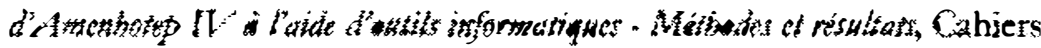
de la Société d'Egyptologie de Gentye, vol. 4, 2 tomes, 243p. 185 planches (Genève 1949).

Sur les différents projets 30 en archélogie consulter les actes des coliomues suivints :

Robert Vergyieux $200 \mathrm{k}$, Edjteut scientifique en collaboration axec C.

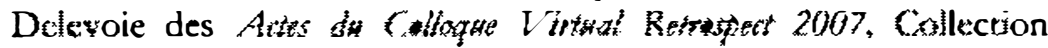
Archéovisiom aux éditions Ausonius, (Bordeaux 200B! 
Robert Vergnieux 2006, Editeur scientifique en collaboration avec C. Delevoie des Actes dus Colloque Virtsal Retrospect 2005, Collection Archéovision aux éditions Ausonius, (Bordcaux 2006).

Robert Vergnieux 2004, Editeur scientifique en collaboration avec C. Delevoie des Actes du Colloque I irtual Retrospect 2003, Collcction Archéovision aux éditions Ausonius, (Bordeaux 2004).

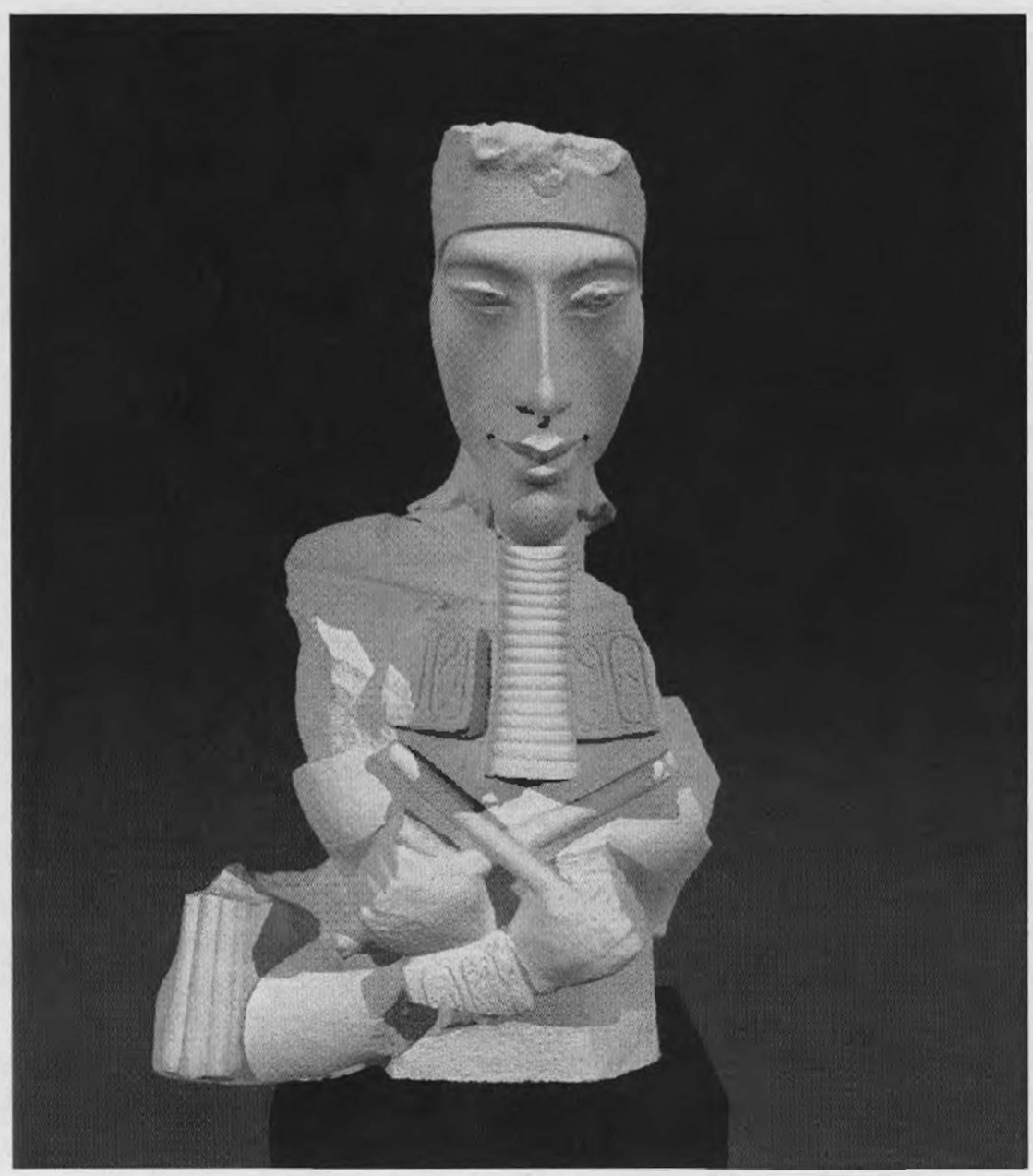

Figure 1: Version numérique (V(O) du bustc d'Akhenaton conservé au Louvte (Archéovision - Archéotransfert) 


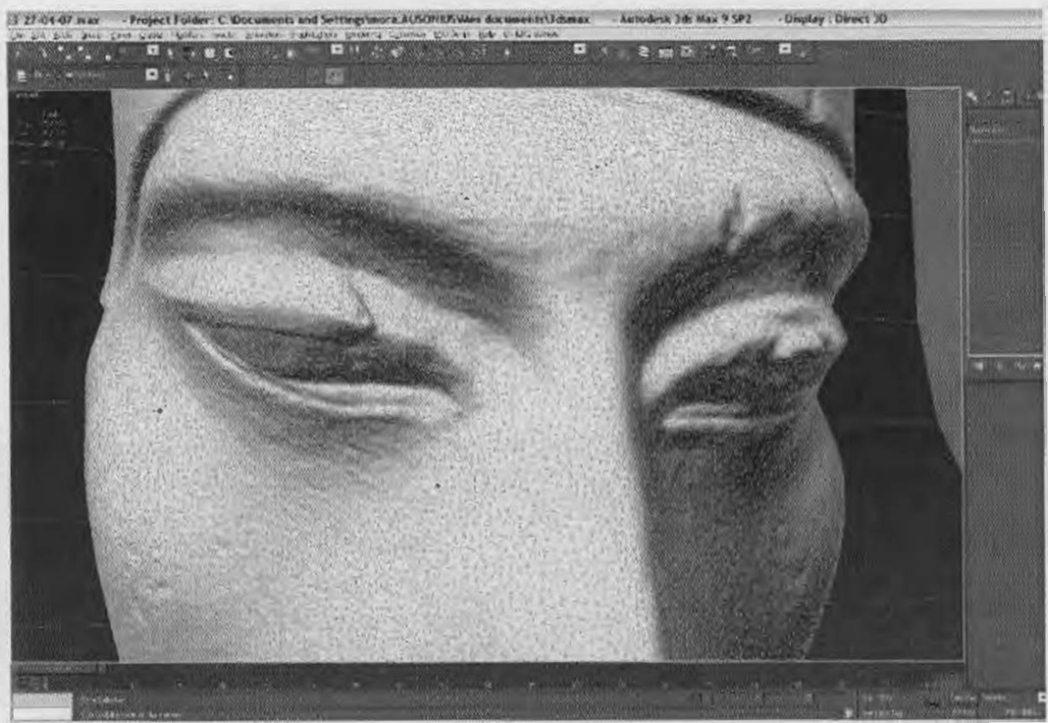

Figure 2: Restaurations virtuelles sur lc buste numérique d'Akhenaton (Archéovision - Archéotransfert)

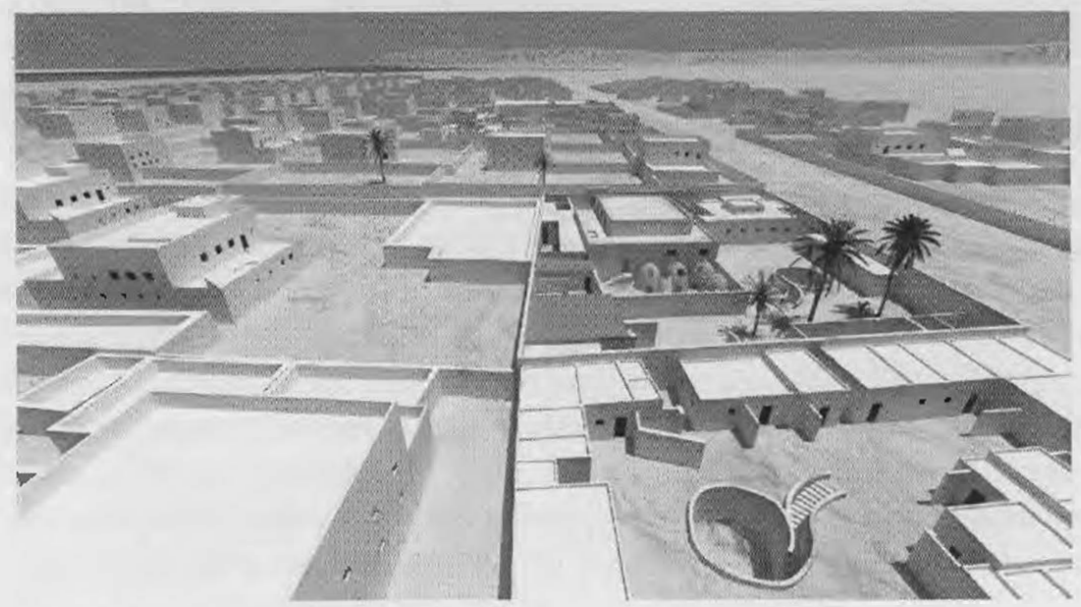

Figure 3: Version V2: Maison de Djehoutymes, aspects extérieurs (Archécovision Archéotransfert-Musée de Genève). 


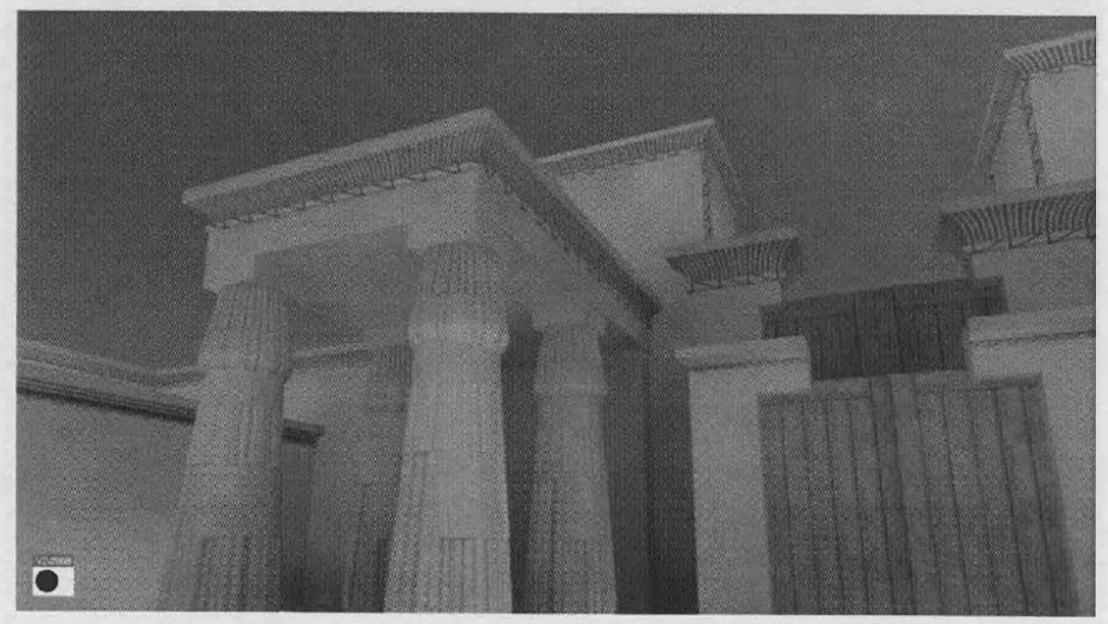

Figure 4: Version V2: Pylône du sanctuaire d'Aton (Archéovision Archéotransfert-Musée de Genève) 
The Compurer Working Group of the International Association of Fegyptologists, usually called by the name Informatique et Fgyptologie, has been in existence since 1983. Its initial existence was primarily driven by the need to devise ways of encoling, representing, and printing hietoglyphs on computers. But fior the past 20 years the group has been focusing the attempts of Egyptologists to find creative and above all useful ways of using information technology to aid in the research and reaching of Ancient Eyypt, and for providing a forum for discussion of these methods. Other than its work with hieroglyphs, the group was responsible for bringing the Internet to the attention of Figyptologists in the mid 1990).

Previous publications of the group, in addirion to conference procecelings, include the Manuel de Coclage (the guide to the encoding of hieroglyphs), Hicroglyphica (an extended library of hieroglyphic signs), and the Multilingual Fegyptological Thesaurus (a standardised set of terms for categorising objects, available in English, Dutch, (jeman, French, Italian, Spanish and Portuguese).

The 2008 mecting rook place in July at the Kunsthistorisches Museum, Vienna, where 16 papers were presented on topics including databases, complex systems, 31) modelling, textual analysis systems, the uses of the intemet for sharing plotographs, and bibliography. This publication provides an essential snapsot of the present uses to which $1 \%$ is placed in the study of Ancient Egypt.

Nigel Strudwick is a curator at the British Museum, who has excavared in Egypt for many vears. His main interests are in the ()ld Kingdom, and in the archacelogy of Thebes. He has been an active member of Informaticue et Figyprologic since 1986, and has chaired the group since 2000 . His IT work has ranged widely; he is most proud of almost single-handedly introducing Egyptology the lnternet in 1994--5.
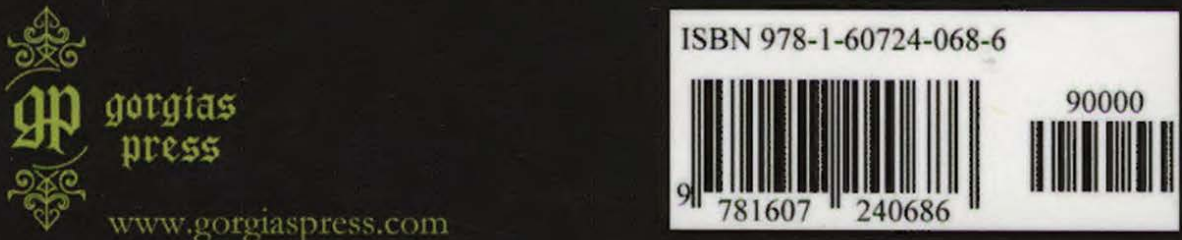